Original Article

\title{
Surgical Volumes in a Regional Trauma Center: Is It Enough?
}

\author{
Mina Lee, Giljae Lee, Jungnam Lee, Byungchul Yu * \\ Department of Trauma Surgery, Gachon University, Gil Medical Center, Incheon, Korea
}

\section{Article history:}

Received: October 2, 2018

Revised: January 17, 2019

Accepted: February 9, 2019

\section{${ }^{*}$ Corresponding Author:}

Byungchul Yu

Department of Trauma Surgery, Gachon

University Gil Medical Center, Incheon,

Korea

E-mail: kane2123@gilhospital.com

ORCID

Mina Lee

https://orcid.org/0000-0002-9725-8993

Giljae Lee

https://orcid.org/0000-0002-7943-5933

Jungnam Lee

https://orcid.org/0000-0002-3877-6600

Byungchul Yu

https://orcid.org/0000-0002-2444-2343

\section{ABSTRACT}

Purpose: This study evaluated the surgical volumes and types of specific surgical procedures in a single trauma center for 3 consecutive years.

Methods: From January 2014 to December 2016 there were 9,530 injury cases in the trauma registry that were reviewed.

Results: There were 1,502 patients (15.8\%) with an injury severity score over 15 , of which $426(28.4 \%)$ underwent an emergency operation or had an interventional radiology procedure. There were 186 craniotomies, 87 laparotomies, and 74 interventional radiology procedures performed.

Conclusion: The number of emergency operations by each dedicated trauma surgeon was very low therefore implementation of an acute-care surgery model is appropriate to consider together with changes to the training program for trauma surgeons.

Keywords: general surgery, trauma centers, wounds and injuries

\section{Introduction}

The mid-1970s to the 1980s was the golden age of trauma surgery; during this era, trauma surgeons frequently performed complex trauma operations including hepatic resection, pancreaticoduodenectomy, and aortic repair [1]. In the same era, trauma surgeons could be the "master surgeon." However, following the golden age of trauma surgery, several critical changes have emerged with improvements in imaging techniques including computed tomography and ultrasonography, and the use of nonoperative management for solid organ injuries. In addition, violence associated with alcohol, drugs and gang warfare has decreased in Korea. Moreover, angioembolization has further enhanced nonoperative management of internal organ bleeding, and development of endovascular stents could treat vascular injuries without surgical repair. By contrast, surgical critical care has emerged as a discipline, and trauma has been absorbed into comprehensive intensive care for all patients with injuries. These changes have led to disinterest in trauma surgery as a career and avoidance of trauma responsibilities by many practicing general surgeons in the United States[2].

In Korea, with the trauma center development plan of the government, trauma surgeons who work in a regional trauma center must be dedicated to trauma surgery only. Some argue that this has caused several problems. Firstly, it has caused disinterest in trauma surgery as a career and has led to a decrease in the number of applicants for trauma surgery fellowships. Secondly, trauma surgeons lack surgical experience, especially those whose fellowship was training in trauma. Thirdly, dedicated specialists for non-trauma emergency surgery are lacking. Lastly, this system could not be maintained without financial support from the government. Implementation of the acute-care surgery model can be an option to solve these problems.

The purpose of this study was to report the surgical volume in a regional trauma center in Korea and review the acute care surgery system in the United States. For this purpose, we analyzed the surgical data recorded by trauma surgeons in a single regional trauma center. 


\section{Materials and Methods}

Gachon University, Gil Medical Center, Incheon, South Korea is an academic hospital with 1,500 beds, and serves a population of 3 million people. The trauma center of this hospital is oneof the first specialist trauma centers established in Korea and its overriding goal was to establish a regionalized Level 1 trauma center. The number of trauma-related admissions is estimated to be $>3,000$ per year, of which 500 to 550 patients have an injury severity score (ISS) $>15$. There are 17 full-time trauma surgeons that currently work at the center, of whom 8 have certification from the general surgery board. There was no change in the number of general surgeons in the study period.

Data of a single institute (Gil Medical Center, Incheon, Korea) from the Korean Trauma Data Bank (KTDB) was extracted. The variables analyzed included age, sex, systolic blood pressure upon arrival at the Emergency Department, Revised Trauma Score, ISS, Glasgow Coma Scale score, transfusion volume, operative procedures, and cause of death. Patients who had undergone either an emergency operation or interventional radiology (IVR), or both, were admitted between January 2014 and December 2016 and reviewed.

The institutional review board at Gil Medical Center waived the need to obtain informed patient consent because only existing materials and documents were used. Data were collected and processed anonymously. Patients were able to refuse the use of their data at any time without any adverse consequences.

\section{Results}

During the study period, there were 9,530 patients with injuries that were registered in the KTDB for the single institution selected to study. Of the 1,502 patients $(15.8 \%)$ with an ISS >15, 426 (28.4\%) had undergone an emergency operation or had IVR. The proportion of the male patients was high (75.4\%), and the patients' mean age was $50.7 \pm 18.2$ years. Most injuries were caused by blunt trauma (94.4\%), and the mean ISS and GCS score was $27.4 \pm 9.0$, and $7.86 \pm 6.57$, respectively. The overall mortality rate of the study group was $20.9 \%$ (Table 1 ). Of the 342 emergency operations (80.3\%), 64 were IVR procedures (15.0\%), and 20 (4.7\%) were hybrid (emergency operation combined with IVR) procedures (Figure 1). The most common emergency operation was a craniotomy. Laparotomies and preperitoneal packing were performed by the general surgeon in 87 and 14 cases, respectively (Figure 2). The annual number of laparotomies and IVR procedures was stable during the study period.
Table 1. Demographics of patients.

\begin{tabular}{lc}
\hline Characteristic & Total $(n=426)$ \\
\hline Male: Female & $321(75.4): 105(24.6)$ \\
\hline Age $(y)$ & $50.7 \pm 18.2$ \\
\hline Blunt trauma & $402(94.4)$ \\
\hline Penetrating trauma & $22(5.2)$ \\
\hline Injury severity score & $27.4 \pm 9.0$ \\
\hline Glasgow Coma Scale & $7.86 \pm 6.57$ \\
\hline Initial systolic blood pressure $(\mathrm{mmHg})$ & $113.3 \pm 46.9$ \\
\hline Initial heart rate (per min) & $88.8 \pm 29.2$ \\
\hline Red blood cell transfusion (units/24 h) & $4.82 \pm 8.45$ \\
\hline Death & $89(20.9)$ \\
\hline
\end{tabular}

Data are presented as $n(\%)$ or mean \pm SD.

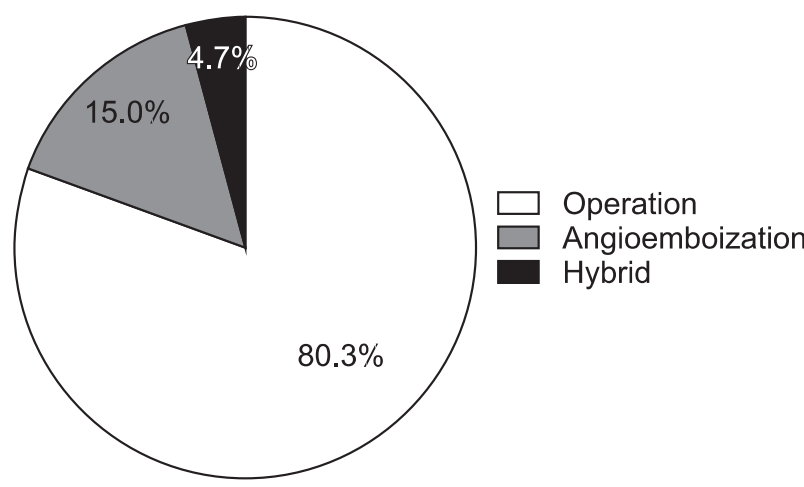

Figure 1. Percentage of interventions.

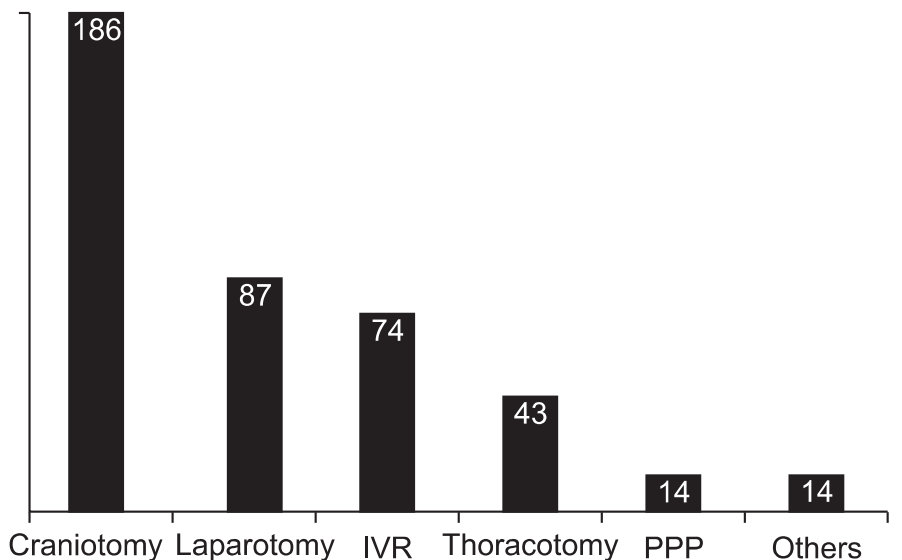

Figure 2. Number of procedures. IVR = interventional radiology; $\mathrm{PPP}=$ pre-peritoneal packing. 


\section{Discussion}

In this study, only $28.4 \%$ of the patients with severe injuries (ISS $>15$ ) received emergency procedures between January 2014 and December 2016. The remaining patients were admitted to a trauma intensive care unit and treated non-operatively. There were 87 emergency laparotomies performed by certified general surgeons $(n=8)$ in the center over 3 years. Each general surgeon carried out on average 3 to 4 laparotomies per year throughout the study periods. Although the work load of critical care and elective trauma laparotomies could not be analyzed in this study, it is thought that this surgical volume is insufficient for surgical training, and maintenance of surgical skills to facilitate job satisfaction.

In the United States, it was reported in 1992, and more recently in 2012, that not only medical students have developed a disinterest in trauma surgery and its responsibilities as a career, but so have general surgeons [2,3]. Thus, trauma surgeon numbers have been falling to a state of crisis. The main causes for disinterest in trauma are the increasingly limited operative exposure and the expanding burden of nonoperative responsibilities assigned to a trauma surgeon [4].

Various options are available to increase operative exposure in the trauma service. A popular trend reported in 2004 was to add non-trauma emergency surgery to trauma surgery responsibilities [5]. This provides increased surgical exposure for trauma surgeons, and at the same time, surgeons who perform elective surgery could be relieved of the burden of these responsibilities. Most non-trauma surgical emergencies consist of draining infected soft tissue and excising necrotic gastrointestinal structures [4]. Thus, acute-care surgeons can be part of the solution. However, it should not be restricted to gastrointestinal surgeries [1].

Some have argued that acute-care surgery, including nontrauma responsibilities, can interfere with a trauma surgeon's ability to care for trauma patients. Previous reports observed that while emergency general surgery procedures were performed throughout the week, trauma cases were more likely to occur over the weekend. Peak operating times were also different for emergency general surgery and trauma cases [6]. Most of the emergency procedures are not an emergency and can be scheduled during regular hours for efficient use of operating rooms. However, no study related to this topic has been conducted in Korea until now.

Patients who require non-trauma emergency operations need young, energetic surgeons who are dedicated to meeting patient needs and advancing the science of emergency surgical care $[7,8]$. Hospitals in the US are facing a crisis in emergency care, whereas the Centers for Disease Control and Prevention analysis of 2006 hospital admissions indicated that $40 \%$ of cases were emergencies and $25 \%$ were urgent [3]. A dedicated and mature emergency general surgery service demonstrated a decrease in mortality and length of stay [9]. Unfortunately, no data are available regarding the outcomes of non-trauma emergency general surgery in Korea.

In summary, trauma surgery, including acute-care surgery and surgical critical care, has several advantages: 1) useful for training and maintaining surgical skills, 2) can be attractive without interference of trauma care, 3) improves outcomes of non-trauma emergency operation, 4) implementation of surgical critical care, and 5) increases the exposure of medical students and residents to surgical attendance. Thus, a training program should be developed, including surgical critical care, trauma surgery, and emergency general surgery $[1,3,10]$.

This study has several limitations. This study reported emergency surgical volume in 1 selected regional trauma center, so the number of elective trauma surgeries, specific operative procedures, critical care volume, bedside procedures, and cost effectiveness were not analyzed. In addition, other regional trauma center's surgical volume cannot be confirmed and could be very different to the regional trauma center selected to review in this study. A nationwide study reviewing surgical volume would overcome these limitations.

\section{Conflicts of Interest}

No potential conflict of interest relevant to this article was reported.

\section{References}

[1] Ciesla DJ, Moore EE, Moore JB, Johnson JL, Cothren CC, Burch JM. The academic trauma center is a model for the future trauma and acute care surgeon. J Trauma 2005;58(4):657-61.

[2] Richardson JD, Miller FB. Will future surgeons be interested in trauma care? Results of a resident survey. J Trauma 1992;32(2):229-33.

[3] Moore HB, Moore PK, Grant AR, Tello TL, Knudson MM, Kornblith LZ, et al. Future of acute care surgery: A perspective from the next generation. J Trauma Acute Care Surg 2012;72(1):94-9.

[4] Moore EE. Trauma surgery: Is it time for a facelift? Ann Surg 2004;240(3) :563-4.

[5] Scherer LA, Battistella FD. Trauma and emergency surgery: An evolutionary direction for trauma surgeons. J Trauma 2004;56(1):7-12.

[6] Frykberg ER, Dennis JW, Bishop K, Laneve L, Alexander RH. The reliability of physical examination in the evaluation of penetrating extremity trauma for vascular injury: Results at one year. J Trauma 1991;31(4):502-11.

[7] Woerth G. IOM and ACS warn of the impending crisis in emergency care: Emergency departments overwhelmed, underfunded, and dangerously fragmented. Bull Am Coll Surg 2007;92(3):20-6.

[8] Sheldon GF. Access to care and the surgeon shortage: American Surgical Association forum. Ann Surg 2010;252(4):582-90.

[9] Diaz JJ Jr, Norris PR, Gunter OL, Collier BR, Riordan WP, Morris JA Jr. Does regionalization of acute care surgery decrease mortality? J Trauma 2011;71(2):442-6.

[10] Koea JB, Srinivasa S, Hundal H. Provision of acute general surgery: A systematic review of models of care. J Trauma Acute Care Surg 2014;76(1) :219-25. 